\title{
mRNA 5'-leader trans-splicing in the chordates
}

\author{
Amanda E. Vandenberghe, ${ }^{1}$ Thomas H. Meedel, ${ }^{2}$ and Kenneth E.M. Hastings ${ }^{1,3}$ \\ ${ }^{1}$ Montreal Neurological Institute and Biology Department, McGill University, Montreal, Quebec, Canada H3A 2B4; \\ ${ }^{2}$ Biology Department, Rhode Island College, Providence, Rhode Island 02908, USA
}

We report the discovery of mRNA 5 -leader trans-splicing (SL trans-splicing) in the chordates. In the ascidian protochordate Ciona intestinalis, the mRNAs of at least seven genes undergo trans-splicing of a 16-nucleotide 5'-leader apparently derived from a 46-nucleotide RNA that shares features with previously characterized splice donor SL RNAs. SL trans-splicing was known previously to occur in several protist and metazoan phyla, however, this is the first report of SL trans-splicing within the deuterostome division of the metazoa. SL trans-splicing is not known to occur in the vertebrates. However, because ascidians are primitive chordates related to vertebrate ancestors, our findings raise the possibility of ancestral SL trans-splicing in the vertebrate lineage.

[Key Words: RNA splicing; SL trans-splicing; chordate/vertebrate evolution]

Received November 7, 2000; revised version accepted December 18, 2000.

mRNA 5'-leader trans-splicing is a mode of gene expression reported in several organisms in which the original $5^{\prime}$ ends of pre-mRNAs are discarded and are replaced by the 5' segment of a spliced leader (SL) RNA (Bonen 1993; Blumenthal 1995; Davis 1996). The function of SL transsplicing is not clear in every case and may vary. Multiple roles have been proposed, including mediation of mRNA stability or translatability (Maroney et al. 1995), resolution of polycistronic pre-mRNAs (Agabian 1990; Blumenthal 1995), and production of functional mRNAs from RNA polymerase I transcripts (Lee and Van der Ploeg 1997). In some organisms, only a subset of mRNAs undergo SL trans-splicing but in others, most or all do (Agabian 1990; Bonen 1993; Davis 1996). SL trans-splicing occurs alongside of the conventional cis-splicing process that removes introns from pre-mRNAs (Bonen 1993; Blumenthal 1995; Mair et al. 2000). There are mechanistic parallels between SL trans-splicing and conventional cis-splicing, including the use of the same set of nucleotide sequence features to mark splice donor and acceptor sites, and a strong resemblance of SL RNAs to spliceosomal U snRNAs (Agabian 1990; Bonen 1993; Nilsen 1993). These similarities imply a close evolutionary relationship between cis-splicing and SL trans-splicing, but the nature of this relationship and the overall evolutionary history of SL trans-splicing are not clear, in part because the phylogenetic distribution of SL transsplicing has not been clearly delineated.

The known phylogenetic distribution of SL trans-

${ }^{3}$ Corresponding author.

E-MAIL khastings@mni.mcgill.ca; FAX (514) 398-1509.

Article and publication are at www.genesdev.org/cgi/doi/10.1101/ gad.865401. splicing is uneven and includes several protist and metazoan groups (Bonen 1993; Blumenthal 1995; Davis 1996). It was first discovered in a protist group, the trypanosomes (Campbell et al. 1984; Kooter et al. 1984; Milhausen et al. 1984), then subsequently in two protosotome metazoan phyla, Nematoda (Krause and Hirsh 1987) and Platyhelminthes (flatworms) (Rajkovic et al. 1990) and in Euglena, a protist distantly related to trypanosomes (Tessier et al. 1991). SL trans-splicing has not been reported in advanced protostome phyla, that is, the arthropods, annelids, or molluscs, nor among the deuterostomes, the great division of the metazoa that includes chordates/vertebrates. However, because each discovery of SL trans-splicing was a fortuitous result of a detailed study of particular genes/mRNAs, and because extensive studies along these lines have been carried out in only a small number of organisms, the true phylogenetic range of SL trans-splicing is unknown.

Here we report the discovery of SL trans-splicing among the deuterostomes, in the ascidian Ciona intestinalis, a chordate. This finding considerably extends the known phylogenetic range of SL trans-splicing. Moreover, because ascidians are primitive chordates related to vertebrate ancestors (Berrill 1955; Katz 1983), SL transsplicing in ascidians raises the possibility of ancestral SL trans-splicing in vertebrate evolution.

\section{Results}

A common 5' sequence on multiple Ciona mRNAs

Evidence for trans-splicing in Ciona emerged during studies of a muscle gene encoding the contractile regulatory protein troponin I (TnI). We determined the com- 
plete sequence of body-wall muscle TnI mRNA by cDNA cloning (MacLean et al. 1997) and 5'-RACE analysis (see Materials and Methods). Surprisingly, database searching revealed that the first 16 nucleotides of the TnI mRNA sequence were similar or identical to the first 16 nucleotides of 3 other Ciona mRNAs (Fig. 1), including the CiMDFa mRNA encoding a MyoD-like muscle transcription factor (Meedel et al. 1997). The existence of a common sequence at the $5^{\prime}$-ends of diverse mRNA species is characteristic of SL trans-splicing (Bonen 1993; Blumenthal 1995; Davis 1996).

Because the various mRNA 5'-end sequences in Figure 1 had been obtained by use of the same $5^{\prime}-\mathrm{RACE}$ technique, we confirmed the presence of the common 5 ' sequence (henceforth called the SL sequence) by an independent method on the basis of RT-PCR amplification with a leftward gene-specific primer for TnI, coupled with a rightward primer containing the SL sequence (SL primer). Products of the expected distinct sizes were produced from alternatively spliced body-wall muscle and heart TnI mRNAs (Fig. 1B). Sequence analysis of the body-wall muscle product showed that the SL primer had primed rightward synthesis on TnI cDNA molecules immediately upstream of mRNA nucleotide 17, exactly as expected on the basis of $5^{\prime}$-RACE sequence data of Figure 1A (data not shown). Similar experiments with CiMDF(MyoD) also generated a body-wall muscle RTPCR product of the predicted size that hybridized with a CiMDF probe (data not shown). These results confirmed independently the presence of the SL sequence established originally by 5'-RACE analysis.

To explore the possibility that additional Ciona mRNAs might contain the 5' SL sequence, we designed an RT-PCR protocol to amplify any poly(A)-containing mRNA that also contained the 5' SL sequence. Reverse transcription of the entire mRNA population was primed by oligo(dT) linked 3' to an arbitrary anchor sequence, and subsequent amplification was based on leftward priming with the anchor sequence and rightward priming with the SL primer. Heterogeneous products ranging from $\sim 0.7$ to $2.5 \mathrm{~kb}$ were produced from bodywall muscle and heart RNA, with prominent bands at $1.6,1.0$, and $0.8 \mathrm{~kb}$ among the body-wall muscle products (Fig. 2A) DNA from gel regions including the 1.0and $0.8-\mathrm{kb}$ bands was recovered and cloned. Sequence analysis of three randomly chosen insert-containing clones revealed three different and apparently complete mRNAs (GenBank AF237689-AF237691). In each case, the presence of $\operatorname{poly}(\mathrm{A})$ at one end, with an AATAAA poly(A) addition signal 20 bp upstream, served to identify the coding strand. In each case, the first ATG triplet on the coding strand was in a Kozak translation initiation consensus (Kozak 1991) context, CANNATG, and initiated an ORF of 190-250 codons encoding a protein related to previously characterized proteins (see legend to Fig. 2). As shown in Figure 2B, in each RT-PCR product, the SL primer sequence was found upstream of the ORF and separated from it by one or more in-frame stop codons. This finding establishes that the SL primer had primed rightward synthesis within the $5^{\prime}$-untranslated mRNA sequence - as expected for priming from the 5 '-end.

Figures 1 and 2, together, show that at least seven Ciona mRNAs contain the 5' SL sequence, and, given the heterodisperse nature of the RT-PCR products in Figure 2A, there may be more.

\section{Gene structure consistent with SL trans-splicing}

Analysis of TnI gene structure provided additional evidence consistent with SL trans-splicing. Upon isolation and sequence analysis of the Ciona TnI gene, we found exons accounting for the entire length of the TnI mRNA except for the first 16 nucleotides. Proceeding upstream from the TnI ATG start codon, the genomic DNA sequence aligned with the mRNA sequence until an AG dinucleotide immediately upstream of mRNA nucleotide 17 , beyond which the sequences diverged sharply
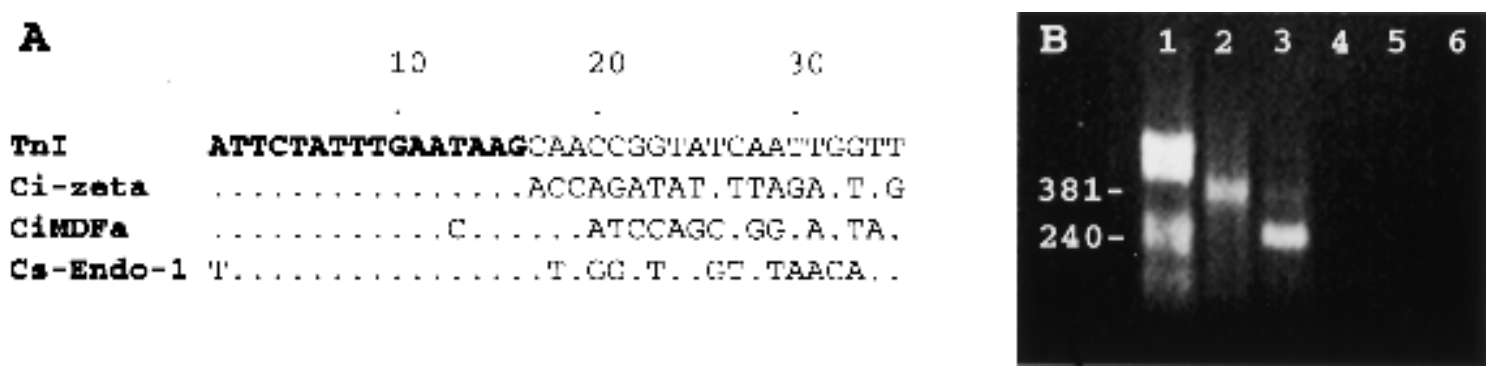

Figure 1. A common $5^{\prime}$-sequence in several Ciona mRNAs. (A) A common 16-nucleotide sequence (bold) at the $5^{\prime}$-end of TnI mRNA, determined by 5 '-RACE, and three additional Ciona mRNAs found by BLAST (Altschul et al. 1997) search of the GenBank database with the TnI 5'-sequence. The first 35 nucleotides of each mRNA are shown; dots indicate identity with the TnI mRNA sequence. Ci-zeta mRNA (GenBank AJ002142) obtained from ovary, encodes a proteasome subunit (Marino et al. 1999). CiMDFa mRNA (Genbank U80079) is expressed in larval tail muscle and adult body-wall muscle (Meedel et al. 1997). Cs-Endo-1 mRNA (GenBank AB024925) is a maternal mRNA from oocytes of Ciona savignyi (Imai et al. 1999). (B) RT-PCR amplification of TnI mRNAs with TnI-specific leftward priming using the SL primer for rightward priming. (Lane 1) Size markers (pBR322 HaeIII digest; top bands 587-434 bp, middle bands 267-184 bp, bottom bands $\leqslant 124$ bp). (Lanes 2,4) Heart RNA template; (lanes 3,5) body-wall muscle RNA template; (lane 6) no RNA. Reverse transcriptase was omitted in lanes 4 and 5. Products of 381 bp (heart) and 240 bp (body-wall muscle) are expected; the size difference reflects tissue-specific alternative RNA splicing (MacLean et al. 1997). 

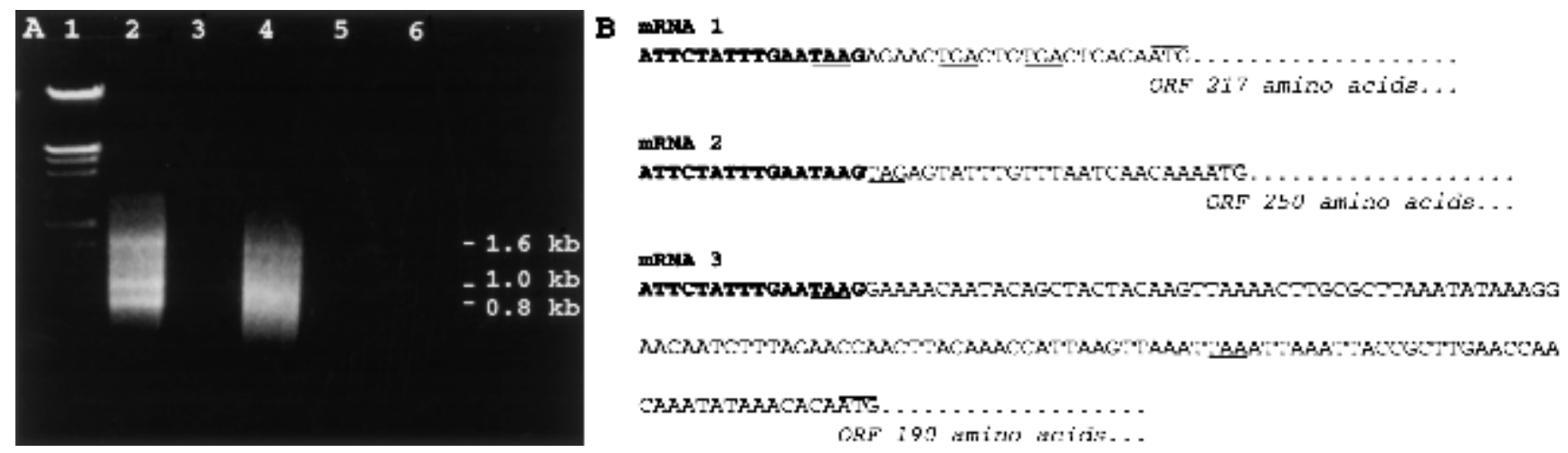

Figure 2. Additional mRNAs containing the SL sequence. (A) Amplification of multiple mRNAs by RT-PCR with SL and oligo(dT)based primers. (Lane 1) Size markers [ $\lambda$ DNA, HindIII and EcoRI digest; in kilobases, from top to bottom, 21, 5.1/5.0, 4.2, 3.5, 2.0/1.9, 1.6, and (not visible) 1.4, 0.95, 0.83, and 0.5]. (Lanes 2,3) Body-wall muscle RNA template; (lanes 4,5) heart RNA template; (lane 6) no RNA. Reverse transcriptase was omitted in lanes 3 and 5. (B) 5'-untranslated sequences of three apparently complete mRNAs (GenBank AF237689--AF237691) recovered by cloning DNA from the 1.0 and 0.8-kb bands in $A$, lane 2. The SL sequence is bolded; not shown is the BamHI site engineered at the $5^{\prime}$-end of the SL primer. The overlined ATG codons initiate ORFs of the lengths indicated, each terminated by a TAA stop codon and followed by an apparently complete 3 '-untranslated sequence. In-frame stop codons within the 5 '-untranslated sequences are underlined. The mRNA 1 ORF encodes a protein resembling HR-29 (Takagi et al. 1993), a myofibrillar protein from body wall muscle of the ascidian Halocynthia roretzi (46\% identity over 207 aligned residues). The mRNA 2 ORF encodes a novel protein containing 17 PTDAVTL repeats resembling the mucin heptad $[\mathrm{PTE} / \mathrm{E} / \mathrm{V})(\mathrm{P} / \mathrm{T}) \mathrm{TV}] \mathrm{repeats}$ of mammalian zonadhesins (Gao and Garbers 1998). The mRNA 3 ORF encodes a protein resembling vertebrate 27-kd heat-shock protein, hsp27 (Cooper and Uoshima 1994) (42\% identity over 177 aligned residues).

(Fig. 3). AG is the near-universal splice acceptor intron/ exon boundary sequence for both cis- and trans-splicing (Bonen 1993; Blumenthal 1995; Davis 1996). Moreover, two additional features of vertebrate splice acceptor sites (Kramer 1996) were also present, that is, an A residue within the branchpoint consensus sequence YNCTRAY located $16 \mathrm{bp}$ upstream of the AG dinucleotide, and an intervening pyrimidine-rich sequence. Thus, the gene structure is consistent with the possibility of trans-splicing of the SL sequence onto mRNA nucleotide 17. Alternatively, the SL sequence could represent a conventional cis-spliced exon located farther upstream. However, sequence analysis of 5429 bp of DNA upstream of the ATG start codon in one TnI allele (GenBank AF237979) and 2067 bp in another (GenBank AF237978) did not reveal the hypothetical 16-bp exon, nor any smaller microexon that might encode any 3 ' part of the missing 16-nucleotide sequence.

The overall genomic DNA organization near the 5'end of the CiMDF(MyoD) gene resembles that of the TnI gene (T.H. Meedel and J. Lee, unpubl.). Relevant similarities include (1) matching of genomic DNA and mRNA sequences upstream of the ATG translation start codon until an AG dinucleotide immediately upstream of mRNA nucleotide 18, and divergence of the sequences $5^{\prime}$ of this point [CiMDF nucleotide 17, numbered as in the cDNA sequence (Meedel et al. 1997), was absent from the genomic DNA sequence, presumably reflecting an allelic polymorphism], (2) presence of a near-consensus branchpoint sequence $16 \mathrm{bp}$ upstream of the AG dinucleotide, and (3) absence of any upstream exon encoding all or part of the SL sequence within at least $1.4 \mathrm{~kb}$ of the ATG initiation codon. Thus, two genes known to produce mRNAs containing the $5^{\prime} \mathrm{SL}$ sequence have structural features consistent with trans-splicing.

\section{Promoter activity of DNA lacking the SL sequence}

Although all of the foregoing data were consistent with trans-splicing, it remained possible that the SL sequence

Figure 3. Comparison of TnI mRNA 5'sequence and corresponding genomic DNA. The first 35 nucleotides of the mRNA are shown; the SL sequence is bolded and a vertical line marks its junction with the rest of the $5^{\prime}$-untranslated sequence. The sequences of two genomic

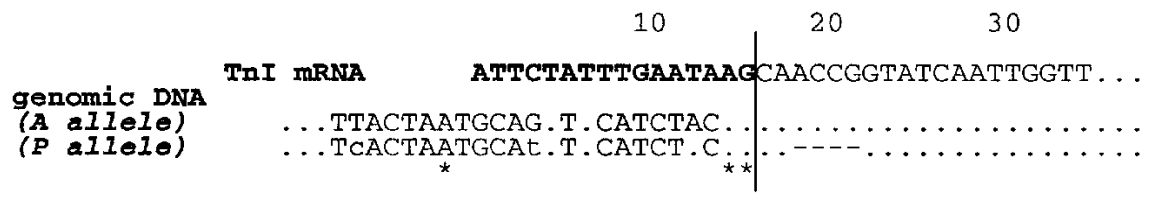

DNA alleles, originating from Atlantic (A allele) or Pacific (P allele) coast animals, are shown. (The mRNA sequence derives from Atlantic coast animals.) Dots in the genomic DNA sequence show identity with the mRNA sequence (except at the left ends, where they signify additional upstream DNA). Differences between the A and P alleles are shown in lower case; a 4-base deletion in the P allele is shown by dashes. The AG dinucleotide present in the genomic DNA at the point of mRNA/genomic DNA sequence divergence is indicated by asterisks adjacent to the vertical line. A third asterisk marks the A residue in the branch point consensus sequence YRCTRAY. 
could be derived from cis-splicing of identical or nearidentical far-upstream exons in the TnI and CiMDF genes and in the other genes that give rise to SL-ended mRNAs. However, we were able to eliminate this hypothesis by showing that far-upstream DNA is not required for muscle-specific expression of a TnI gene construct, nor for the generation of mRNA transcripts containing the SL sequence. We prepared a Ciona TnI $\beta$-galactosidase ( $\beta$-gal) reporter gene construct, CiTnILacZ $(-1.5)$, driven by 1454 bp of DNA upstream of the ATG initiation codon, in which promoterless $\beta$-galcoding sequences were ligated to the $5^{\prime}$-untranslated sequence 23 nucleotides upstream of the ATG start codon (corresponds to nucleotide 58 of the TnI mRNA) (see Materials and Methods). It is important to note that this construct does not include any DNA encoding the SL sequence. Following electroporation (Corbo et al. 1997) into Ciona intestinalis zygotes, development was permitted for $12 \mathrm{~h}$, at which time the embryos are at the tailbud stage and have elaborated specialized structures and cell types including the tail with its notochord and flanking rows of muscle cells. Embryos were fixed and stained to reveal $\beta$-gal expression. We found that the CiTnILacZ $(-1.5)$ construct directed $\beta$-gal expression specifically in tail muscle cells (Fig. 4A) in a high percentage, $>50 \%$, of normally developing electroporated embryos. A control reporter construct, otherwise identical but lacking the Ciona genomic DNA segment, showed no detectable expression. Other studies have established that DNA constructs carrying diverse tissue-specific transcriptional control elements show appropriate celltype-specific expression following electroporation into Ciona embryos (Corbo et al. 1997). These results indicate the presence of a functional muscle-specific promoter within the $\sim 1.5-\mathrm{kb}$ cloned segment of the TnI gene, despite the absence of any sequence corresponding to the first 16 bases (the SL sequence) of the mature TnI mRNA. Effective muscle-specific expression was also obtained with a $\beta$-gal construct containing $1.4 \mathrm{~kb}$ of upstream DNA from the CiMDF gene, likewise lacking the SL sequence (T.H. Meedel and J. Lee, unpubl.). These results are consistent with trans-splicing of the SL se-

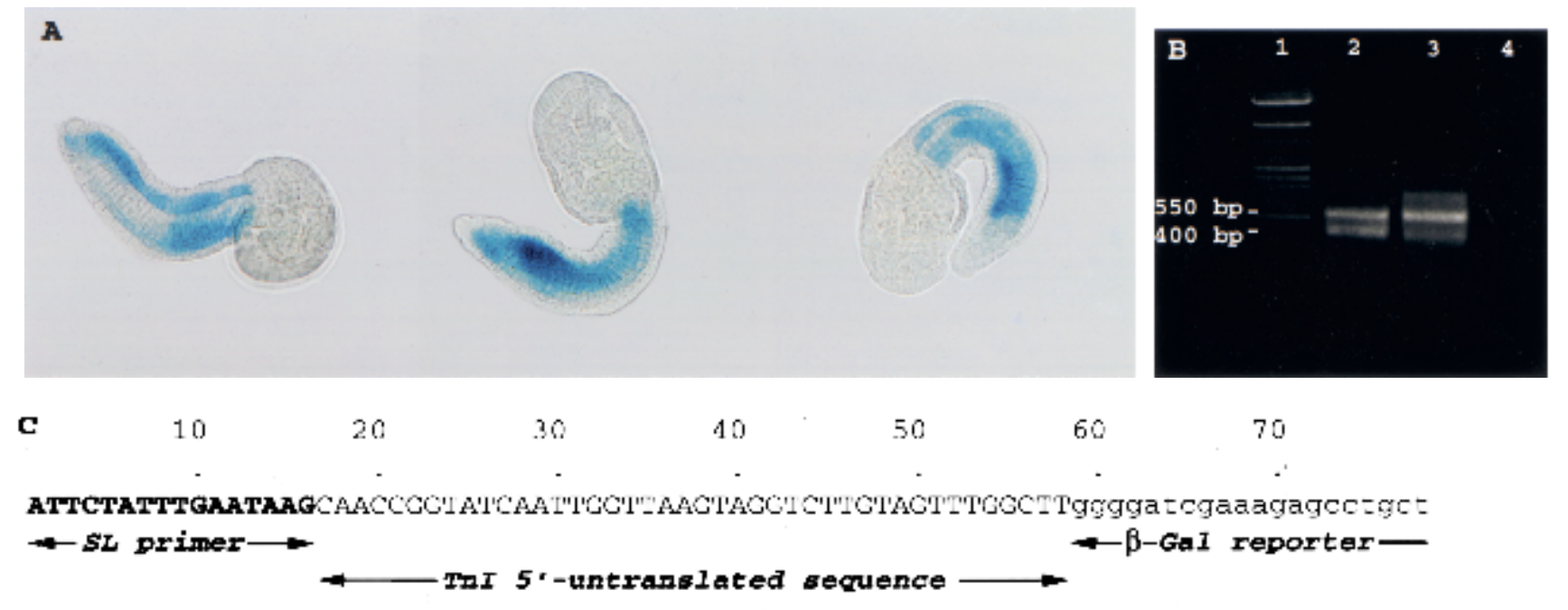

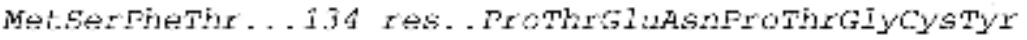

aaigcaaaaagaigtcaccatgtogtttact. . . 402 bp . . cccacggagaatccgacgggttgttact

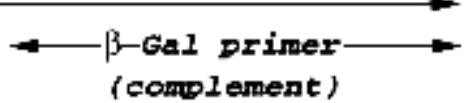

Figure 4. In vivo expression and SL trans-splicing of a chimeric TnI/ $\beta$-gal mRNA from a TnI/ $\beta$-gal gene construct lacking the SL sequence. (A) Expression of $\beta$-gal, revealed by X-Gal staining (blue), in tail muscle of embryos $12 \mathrm{~h}$ following introduction of CiTnILacZ(-1.5) DNA into zygotes by electroporation. (B) RT-PCR amplification of $\beta$-gal mRNA with $\beta$-gal-specific leftward priming, and rightward priming with the SL primer. (Lane 1) Size markers (MBI Fermentas ladder mix, 10-0.1 kb; top visible band, $3 \mathrm{~kb}$, bottom visible band $500 \mathrm{bp}$ ). (Lanes 2,3) RT-PCR products from two different batches of transfected embryos. The template in lane 4 was tRNA, used as a carrier in embryo RNA isolations. A 550-bp product, the size predicted for SL-ended $\beta$-gal mRNA, was produced from both embryo batches. [Production of this product required the presence of both primers and did not occur when CiTnILacZ(-1.5) plasmid DNA was used as the amplification template. An additional product of $\sim 400 \mathrm{bp}$ seen in lanes 2 and 3 required only the $\beta$-gal-specific primer and was produced in control amplifications of CiTnILacZ(-1.5) plasmid DNA; it apparently results from rightward mis-priming by the $\beta$-gal-specific primer upstream of its normal leftward priming site.] (C) DNA sequence of 550-bp RT-PCR product. The 550-bp product (as in $B$ ) was recovered and sequenced using the SL primer (right) and $\beta$-gal-specific primer (left). The leftward sequence confirmed the presence of the SL primer (bold) immediately upstream of TnI mRNA nucleotide 17 . Not shown is the BamHI site engineered at the $5^{\prime}$-end of the SL primer. Sequences deriving from the $\beta$-gal reporter gene are shown in lower case. 
quence, but are not compatible with the hypothesis of cis-splicing of a far-upstream exon. Under a cis-splicing regime, DNA constructs that completely lack the first exon and upstream DNA of a gene would also lack the promoter (which is at the $5^{\prime}$-end of the first exon), yet both TnI and CiMDF genes contain functional musclespecific promoters within DNA segments that lack the first exon (the SL sequence).

\section{Trans-splicing of TnI/ $\beta$-gal chimeric transcripts}

Expression of the CiTnILacZ(-1.5) construct in vivo allowed us to show trans-splicing of an artificial mRNA substrate. Because it contains the TnI mRNA transsplice acceptor site at nucleotide 17 , the TnI/ $\beta$-gal chimeric mRNA transcribed from the CiTnILacZ(-1.5) DNA construct could be subject to the same trans-splicing reaction as is the natural TnI mRNA. Therefore, we used an RT-PCR approach to ask whether $\beta$-gal mRNA molecules expressed in transfected embryos contained the SL sequence. RNA extracted from transfected embryos was reverse transcribed with a $\beta$-gal-specific primer and PCR amplification was carried out with the $\beta$-gal primer for leftward synthesis and the SL primer for rightward synthesis. A product of the expected size $(550$ bp) was obtained (Fig. 4B), and sequence analysis revealed exactly the structure predicted (Fig. 4C). $\beta$-gal sequences were found linked to the TnI 5'-untranslated sequence at nucleotide 58 , and the SL primer was present in the sequence and located immediately upstream of TnI mRNA nucleotide 17. [In control PCR experiments, amplification of CiTnILacZ(-1.5) plasmid DNA with the same primers did not produce the 550-bp product, showing that the SL primer does not coincidentally misprime on the unrelated sequence present in the cloned TnI genomic DNA immediately upstream of nucleotide 17.] The presence of the SL sequence in mRNA transcribed from a gene construct that does not itself encode that sequence is a convincing demonstration of trans-splicing

\section{A Ciona SL-likeRNA}

The occurrence of SL trans-splicing implies a splice donor SL RNA. To characterize Ciona SL RNA, we prepared body-wall muscle RNA by a procedure that did not involve salt precipitation, as this step in our standard RNA isolation procedure removes most small RNAs, and all known SL RNAs are small ( $<150$ nucleotides). Northern blot analysis revealed a $50 \pm 15$ nucleotide RNA species, migrating ahead of the tRNA band, that hybridized with an oligonucleotide complementary to the SL sequence (Fig. 5, lane 3) No similar hybridization was seen in vertebrate (quail) muscle RNA prepared in a similar fashion (Fig. 5, lane 4), or in salt-precipitated Ciona body-wall muscle RNA (lane 5). In comparison with the hybridization signal generated by known amounts of SL-containing RNAs produced by in vitro transcription of cloned mRNA 3 (see Fig. 2), the abundance of the $\sim 50$-nucleotide RNA was estimated to be on the order of $0.1 \%$ of the total RNA mass, representing a severalfold molar excess over the total mRNA population.

The candidate SL RNA was cloned in an approach based on in vitro polyadenylation with poly(A) polymerase (Tessier et al. 1991), followed by RT-PCR amplification using the same primers we used to amplify SL-containing mRNAs in Figure 2. A single abundant RT-PCR product of $\sim 100 \mathrm{bp}$ was generated, and cloning and sequence analysis showed that the majority of the molecules (4/5) represented a single 46-nucleotide RNA species, consistent with the $\sim 50$-nucleotide size of the candidate SL RNA /the polyadenylation/amplification process makes the PCR product 44 nucleotides longer than the original template RNA). The sequence (Fig. 6A) shows the principal feature expected of an SL RNA, that is, the presence of the intron $5^{\prime}$ boundary dinucleotide GU immediately following the SL sequence. The 46nucleotide RNA does not show extensive similarity to known SL RNA sequences, however, previous studies have shown that SL RNAs from different phyla have little direct sequence similarity (Bruzik et al. 1988; Davis
Figure 5. Northern blot detection of Ciona SL RNA. (A) Fluorescence of ethidium bromide stained gel. (B) Autoradiography following transfer to nylon membrane and hybridization with a $5^{\prime}-{ }^{32} \mathrm{P}$-labeled oligonucleotide complementary to the SL sequence. (Lanes 1,6) An RNA marker set (100-1000 nucleotide sizes indicated), to which has been added either $50 \mathrm{ng}$ (lane 1) or $5 \mathrm{ng}$ (lane 6) each of 126-nucleotide, and 666-nucleotide SL-containing in vitro transcripts of a plasmid encoding mRNA 3 (see Fig 2). (Lane 2) Blank; (lane 3) Ciona body-wall muscle RNA (not salt precipitated); (lane 4) quail muscle RNA (not salt precipitated); (lane 5) Ciona bodywall muscle RNA (salt precipitated). Large and small subunit rRNA and tRNA bands are indicated in $A$. Because the samples had not been salt precipitated, lanes 3 and 4 also contain genomic DNA (near sample wells).
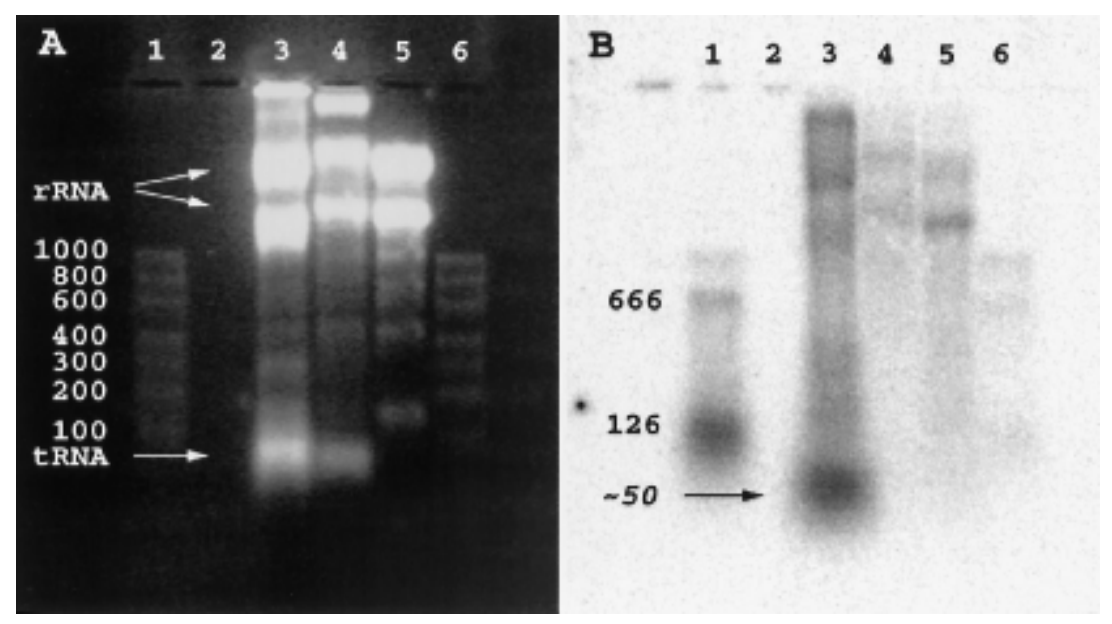
A

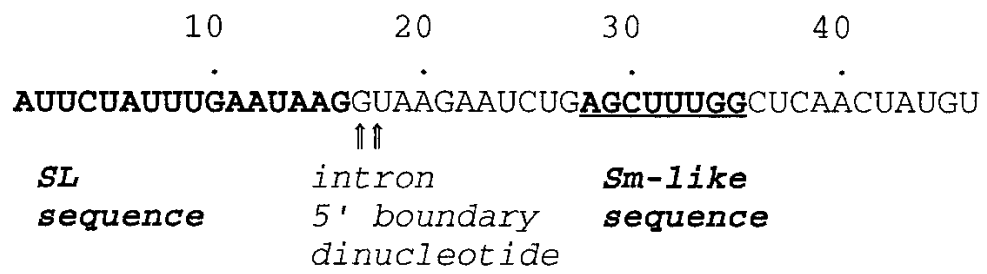

B

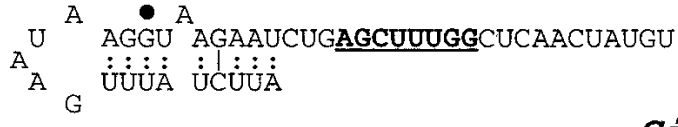

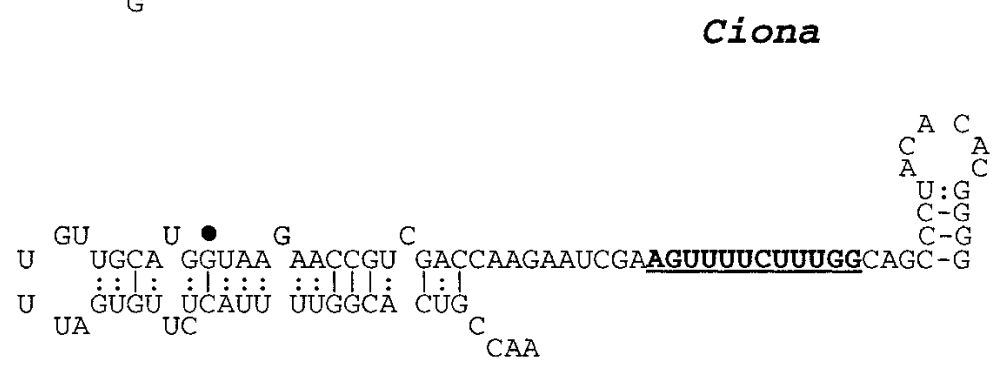

Schistosoma

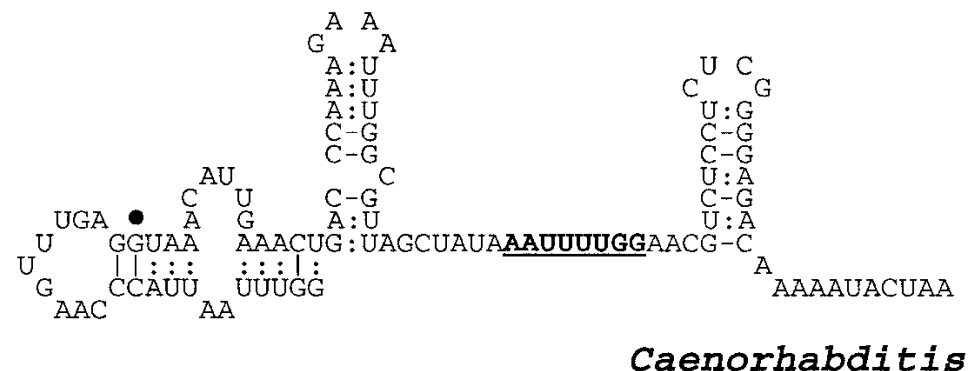

Figure 6. Sequence of Ciona SL RNA and predicted secondary structure comparison with SL RNAs of the flatworm Schistosoma and nematode Caenorhabditis. (A) Sequence of the 46nucleotide Ciona SL RNA. 3'-poly(A), added before amplification and cloning, has been removed from the sequence; the original RNA may have contained one or more A residues at the 3 '-end. The SL sequence is bolded and the Sm-like sequence is underlined and bolded [the canonical Sm consensus is RA(U) $\left.{ }_{\mathrm{n}} \mathrm{GR}\right]$. Arrows mark the intron $5^{\prime}$-boundary GU dinucleotide. (B) Predicted secondary structures. The Ciona SL RNA structure was generated by mfold 3.0 (Mathews et al. 1999) and the Schistosoma and Caenorhabditis structures are from Davis (1996); in all structures the Sm-like sequence (bold, underlined) was constrained to be single stranded. In terms of $\Delta \mathrm{G}$, the Ciona structure shown was among the top two or three generated by calculations based on $22^{\circ} \mathrm{C}$ or $37^{\circ} \mathrm{C}$ and was within $1 \mathrm{kcal} / \mathrm{mole}$ of the optimal solution. Three-H-bond base pairs (i.e., $\mathrm{G}-\mathrm{C}$ ) are indicated by lines, and 2-H-bond base pairs (i.e., $\mathrm{A}-\mathrm{U}$ and $\mathrm{G}-\mathrm{U})$ are indicated by paired dots. A single large dot marks the first $G$ of the intron moiety.
1996). Nonetheless, SL RNAs from different phyla do share several features. In all known SL RNAs, the intron 5' boundary GU dinucleotide is preceded by G and, except for the minor SL RNAs of Caenorhabditis (Ross et al. 1995), followed by A. Moreover, the exon/intron boundary is universally associated with a predicted secondary structure in which both $\mathrm{G}$ residues are involved in the downstream leg of a stem-loop structure near the 5 '-end of the RNA (Bruzik et al. 1988; Rajkovic et al. 1990; Davis 1996). As shown in Figure 6, the Ciona 46nucleotide RNA contains the conserved G/GUA boundary sequence (in a longer sequence G/GUAAGAA shared with the SL RNA of the flatworm Schistosoma), and is present in a predicted stem-loop structure similar to that predicted for other SL RNAs. An additional feature shared by the Ciona 46-nucleotide RNA and all known SL RNAs (Bruzik et al. 1988; Rajkovic et al. 1990; Davis $1996)$ is the presence within the intron moiety of a sequence resembling the consensus-binding site for $\mathrm{Sm}$ proteins (bold and underlined in Fig. 6).

Previously characterized SL RNAs are longer (90-140 nucleotides) than the Ciona 46-nucleotide RNA in both exon (22-51 vs. 16 nucleotides) and intron (54-109 vs. 30 nucleotides) moieties, and contain a predicted stem-loop structure downstream of the Sm sequence (Bruzik et al. 1988; Rajkovic et al. 1990; Davis 1996). In many, but not all cases, another stem-loop is present within the intron upstream of the Sm sequence (e.g., Caenorhabditis SL1 in Fig. 6B). The functional importance of the predicted intron stem-loops is not clear. Chemical modification studies of nematode SL RNA indicate a functional role in trans-splicing for purines in the upstream but not the downstream stem-loop (Hannon et al. 1992), however, the SL RNA of Schistosoma does not contain a similar upstream stem-loop (Rajkovic et al. 1990). Stem-loops may quantitatively facilitate Sm protein binding to $\mathrm{U}$ snRNAs (Jarmalowski and Mattaj 1993; Hinz et al. 1996) but they are not essential (Raker et al. 1999). As Figure 6 shows, the 46-nucleotide Ciona SL RNA is more like the relatively simple SL RNA of Schistosoma than the more complex classical SL RNA secondary structure represented by Caenorhabditis SL1.

\section{Discussion}

Our results establish the occurrence of mRNA $5^{\prime}$-leader (SL) trans-splicing in the ascidian chordate Ciona intestinalis and identify a probable SL RNA splice donor. 
The discovery of SL trans-splicing in the deuterostomes, a major division of the metazoa, shows that SL trans-splicing has a considerably broader phylogenetic range than was known from previous studies (Bonen 1993; Davis 1996). This, and a recent report concerning the class Cestoda within the Platyhelminthes (Brehm et al. 2000), are the first reports to extend the known range of SL trans-splicing in almost a decade (Tessier et al. 1991).

\section{Evidence for SL trans-splicing in Ciona}

We found that at least seven different Ciona mRNAs, including TnI and CiMDF(MyoD) mRNAs, contain a short common sequence at the $5^{\prime}$-ends - a hallmark of SL trans-splicing. Moreover, the TnI and CiMDF(myoD) genes were found to have the two structural features expected of SL trans-spliced genes; (1) the SL sequence itself was not present in the gene, and (2) the mRNA sequence immediately adjacent to the SL sequence was found to correspond to the beginning of an exon, with the characteristic genomic features of a splice acceptor site. In addition, TnI and $\operatorname{CiMDF}(\mathrm{MyoD}) \beta$-gal reporter constructs showed effective muscle-specific expression in embryo transfection experiments, indicating the presence of functional tissue-specific promoters, despite the absence of the SL sequence. Moreover, we demonstrated the presence, in transfected embryos, of TnI/ $\beta$-gal chimeric mRNAs that contain the SL sequence spliced onto the expected splice acceptor site in the TnI $5^{\prime}$-untranslated sequence. Finally, we identified a 46-nucleotide RNA in Ciona body-wall muscle having the expected properties of the predicted splice donor SL RNA.

\section{Extent and role of ascidian SL trans-splicing}

We do not know whether all, or only a subset, of Ciona genes/mRNAs are subject to SL trans-splicing; the seven trans-spliced mRNAs we have identified are a minimum number. These mRNAs are collectively expressed in a wide range of tissues and developmental stages. The biological role of SL trans-splicing in ascidians is currently unknown; proposals for other organisms include mediation of mRNA stability or translatability (Maroney et al. 1995), resolution of polycistronic pre-mRNAs (Agabian 1990; Blumenthal 1995), and production of functional mRNAs from RNA polymerase I transcripts (Lee and Van der Ploeg 1997).

\section{Evolutionary implications}

The occurrence of SL trans-splicing in both protists and metazoa suggests either that it is an ancestral eukaryotic character or that it has arisen independently in multiple organismal lineages (Bonen 1993). Our discovery of SL trans-splicing in a new division of the metazoa favors the ancestral-character hypothesis, because as the number of phyla known to carry out SL trans-splicing increases, the independent-origins hypothesis becomes increasingly less parsimonious. The shared features of known SL RNAs, including the Ciona 46-nucleotide RNA, are consistent with the possibility of descent from a common ancestral RNA, presumably an Sm-binding U snRNA (Bruzik et al. 1988), however, this would not preclude the possibility that the same or similar snRNAs might have independently acquired a trans-splicing role in different organismal lineages. Detailed studies of the trans-splicing mechanism in each group may be required to establish whether SL trans-splicing is an orthologous process throughout its phylogenetic range.

Because ascidians are thought to be related to vertebrate ancestors (Berrill 1955; Katz 1983), our findings raise the possibility that vertebrates could be descended from organisms that carried out SL trans-splicing. In extant vertebrates, SL trans-splicing is unknown, although other forms of trans-splicing have been reported, including the specific joining of particular pre-mRNAs (Li et al. 1999), and exon duplication by trans-splicing between two RNA molecules transcribed from the same gene (Caudevilla et al. 1998; Akopian et al. 1999). However, mammalian cells are able to trans-splice Caenorhabditis SL RNA (Bruzik and Maniatis 1992), which is consistent with the idea of vertebrate descent from SL trans-splicing ancestors, and also suggests the possibility of ongoing vertebrate SL trans-splicing that has not yet been discovered. Nonetheless, given the intense genetic research effort in vertebrates in comparison with ascidians, to have escaped detection implies that vertebrate SL trans-splicing likely occurs to a lesser extent, if at all.

Ascidian TnI and CiMDF(MyoD) genes, among others, are trans-spliced, but extensive studies of the vertebrate $\mathrm{TnI}$ and $M y o D$ gene families, including detailed characterization of transcriptional start sites (Baldwin et al. 1985; Edmondson et al. 1992; Hinterberger et al. 1992; Tapscott et al. 1992; Ausoni et al. 1994; Corin et al. 1994) have revealed no SL trans-splicing, but only the conventional default mechanism of mRNA 5'-end formation. Thus, TnI and MyoD genes have either lost SL transsplicing during vertebrate evolution, or acquired it during ascidian evolution. Functional studies indicate that, given the presence of SL RNA, it is a necessary and sufficient condition for SL trans-splicing that the target premRNA contain a splice acceptor site upstream of the 5'-most splice donor site (Conrad et al. 1991, 1993). It follows that, during evolution, SL trans-splicing of a premRNA could be lost either by mutational loss of the trans-splice acceptor site or by acquisition of a new splice donor site upstream of it in the transcript. The latter event would create a conventional cis-spliced intron within the gene's $5^{\prime}$-untranslated sequence. In view of their possible derivation from SL trans-spliced ancestral genes, it is of interest that vertebrate TnIfast and TnIslow genes contain such introns (Baldwin et al. 1985; Corin et al. 1994). Although other scenarios could also account for the evolution of $5^{\prime}$-untranslated introns, the active possibility of ancestral SL trans-splicing increases the range of evolutionary hypotheses that can be considered in this, and perhaps other aspects of vertebrate gene structure/organization. 


\section{Materials and methods}

\section{Animals and DNA/RNA preparation}

All nucleic acid materials were derived from animals collected at the Sandwich Marina (Cape Cod, MA) except for a $\lambda$ phage genomic DNA library kindly provided by R. Zeller (University of California, San Diego; see below), which was produced from animals collected in coastal southern California. Collection, maintenance of animals, and fertilization and embryonic development were as described (Meedel et al. 1997). Standard preparation of high molecular weight (salt precipitated) RNA, and of genomic (sperm) DNA were as described (Meedel and Hastings 1993). To prepare non-salt-precipitated RNA the salt-precipitation step was omitted.

\section{5'-RACE analysis of TnI mRNA}

The $5^{\prime}$-end of TnI mRNA was amplified from heart and bodywall muscle RNA by use of the Clontech AmpliFinder 5'RACE kit, with TCGGCAGAGATCCATGA and AGTGGATCCGC TGAGTGGCTCAAGTCGTTGGCT as the gene-specific leftward reverse transcription and amplification primers, respectively. This amplified 427-bp (from heart RNA) or 286-bp (from body-wall muscle RNA) products that were gel recovered and subjected to cycle sequencing. Both products had identical 5'untranslated sequences, including the first 16 nucleotidess at the $5^{\prime}$-end.

\section{RT-PCR analysis}

Gene-specific leftward priming on TnI, CiMDF, and $\beta$-gal mRNAs was with GCTCAAGTCGTTGGCTTAG, ACTCAT TCCCGATCCGAAACTC, and AGTAACAACCCGTCGGAT TCTCC, respectively, and the SL primer GGATCCGATTC TATTTGAATAAG was used for rightward priming. For generic amplification of poly $(\mathrm{A})^{+}$mRNAs containing the SL sequence, reverse transcription was primed with an arbitrary T-tailed oligonucleotide GAATTCTACCTCAGAGGAGTCATATTTTTT TTTTTTT. Leftward PCR priming was done with the same sequence, but lacking 12 of the 13 T-residues at the 3 '-end, and rightward priming was done with the SL primer. Gel bands were recovered, cloned in the pCR2.1 T/A vector (Invitrogen), and three randomly chosen clones were sequenced on both strands.

\section{Cloning and analysis of TnI genomic DNA}

Two approaches were used to isolate TnI genomic DNA upstream of that isolated previously (MacLean et al. 1997). Inverse PCR [IPCR, (Ochman et al. 1988)] using TnI gene-specific primers produced a $2.8 \mathrm{-kb}$ product containing $2067 \mathrm{bp}$ of TnI DNA upstream of the ATG initiation codon (P. Pannunzio and K.E.M. Hastings, unpubl.). The IPCR product was subcloned and sequenced and its validity confirmed by genomic DNA amplification between the farthest upstream sequence and previously known TnI sequence downstream of the ATG start codon. Additional upstream DNA was obtained by hybridization screening of a $\lambda$ ZAP Express (Stratagene) phage library (from Robert Zeller) of partial Sau3A-cut Ciona intestinalis DNA using the IPCR product as probe. Several TnI genomic clones were obtained, and one that included 5429 bp of DNA upstream of the ATG codon was completely sequenced on both strands. The genomic phage clone and IPCR product sequences clearly corresponded throughout their $\sim 2$-kb overlap, although allelic differences, which are common in Ciona genes (Meedel and Hastings 1993; MacLean et al. 1997; Meedel et al. 1997) were evident [the IPCR and phage-cloned sequences derive from Atlantic (Cape Cod) and Pacific (southern California) coast animals, respectively].

\section{Introduction of $\beta$-gal reporter constructs into embryos}

CiTnILacZ $(-1.5)$ contains TnI DNA from the subcloned IPCR product (see above) extending from a KpnI site to a Bpu1102I site, 1454 and $23 \mathrm{bp}$ upstream of the ATG start codon, respectively. The blunted Bpu1102I site was ligated to the SmaI end of a 5-kb SmaI/EcoRI fragment from pRSVZ (MacGregor et al. 1987), which contains 7 bp of linker sequence, 38 bp of $5^{\prime}$ untranslated sequence, and the first 31 codons from Drosophila alcohol dehydrogenase mRNA, linked to Escherichia coli LacZ $\beta$-gal sequences and SV40 splicing and polyadenylation sites. Introduction of DNA constructs into Ciona intestinalis zygotes was by electroporation (Corbo et al. 1997) and following development at $18^{\circ} \mathrm{C}$ for $12 \mathrm{~h}$ embryos were fixed and stained with $\mathrm{X}-\mathrm{Gal}$. For RNA isolation, batches of electroporated embryos were examined and sorted at $12 \mathrm{~h}$ and pools of $\sim 25$ normally developing embryos were frozen in $0.77 \mathrm{M}$ mannitol with $20 \mathrm{ug}$ of yeast tRNA. After thawing, RNA was recovered by phenol/ chloroform extraction and ethanol precipitation and samples representing one-eighth of the total yield from a batch of embryos were reverse-transcribed with the $\beta$-gal-specific primer (see above) prior to PCR amplification with the $\beta$-gal primer and the SL primer.

\section{Northern blot analysis}

RNA samples separated by electrophoresis on $2 \%$ agarose gels (Thomas 1980) were vacuum-blotted to Zeta-Probe membrane (BioRad) and hybridized and washed at $37^{\circ} \mathrm{C}$ [as in Rajkovic et al. (1990)] with ACCTTATTCAAATAGAAT (complementary to SL sequence and adjacent intron $5^{\prime}$ boundary dinucleotide) labeled previously with T4 polynucleotide kinase and $\left[\gamma^{32} \mathrm{P}\right]$ ATP. Autoradiography was by PhosphorImaging. RNA markers were the MBI Fermentas low-molecular weight RNA marker set. SL-containing RNAs of 126 and 666 nucleotides were produced by T7 RNA polymerase transcription of plasmid containing the mRNA 3 insert (see Fig. 2) in pCR2.1, previously digested with Hin6I or HincII, respectively.

Sequence analysis of SL RNA

Non-salt-precipitated body-wall muscle RNA ( $3 \mu \mathrm{g})$ was 3' polyadenylated with 3.5 units of poly(A) polymerase (Life Technologies) in $40-\mu \mathrm{l}$ reactions containing $225 \mathrm{mM} \mathrm{NaCl}, 50 \mathrm{mM}$ TrisHCl (pH 8), $10 \mathrm{mM} \mathrm{MgCl} 2,2.5 \mathrm{mM} \mathrm{MnCl}_{2}, 0.25 \mathrm{mM}$ ATP, and $0.5 \mathrm{mg} / \mathrm{ml} \mathrm{BSA}$ for $30 \mathrm{~min}$ at $37^{\circ} \mathrm{C}$. The polyadenylated RNA was subjected to RT-PCR as described above (see, RTPCR analysis) and the single abundant product, $\sim 100 \mathrm{bp}$, was recovered following agarose gel electrophoresis and cloned into pCR2.1. Five clones were picked randomly and sequenced, and four corresponded to the 46-nucleotide SL RNA sequence shown in Figure 6; there were no base substitutions, although 3 '-truncations of 2 ( 2 clones) and 4 nucleotides (1 clone) were observed. The fifth clone contained an unrelated sequence. Parallel analysis of five clones derived from trace amounts of similar-sized RT-PCR products made from RNA that had not been polyadenylated gave a heterogeneous collection of five different sequences.

\section{Acknowledgments}

We thank Robert Zeller for providing animals and a genomic DNA library, Pierre Pannunzio for his contribution to IPCR 
product isolation, and Jamie and Nancy Lee for their hospitality while T.H.M. was Visiting Scientist at the Mayo Clinic, Scottsdale, Arizona. K.E.M.H. is a Killam Scholar of the Montreal Neurological Institute. Research on muscle genes in the authors' laboratories was supported by grants from the Natural Sciences and Engineering Research Council of Canada, the Medical Research Council/Canadian Institutes of Health Research, and Rhode Island College.

The publication costs of this article were defrayed in part by payment of page charges. This article must therefore be hereby marked "advertisement" in accordance with 18 USC section 1734 solely to indicate this fact.

\section{References}

Agabian, N. 1990. Trans splicing of nuclear pre-mRNAs. Cell 61: $1157-1160$.

Akopian, A.N., Okuse, K., Souslova, V., England, S., Ogata, N., and Wood, J.N. 1999. Trans-splicing of a voltage-gated sodium channel is regulated by nerve growth factor. FEBS Lett. 445: $177-182$.

Altschul, S.F., Madden, T.L., Schaffer, A.A., Zhang, J., Zhang, Z., Miller, W., and Lipman, D.J. 1997. Gapped BLAST and PSI-BLAST: A new generation of protein database search programs. Nucleic Acids Res. 25: 3389-3402.

Ausoni, S., Campione, M., Picard, A., Moretti, P., Vitadello, M., De Nardi, C., and Schiaffino, S. 1994. Structure and regulation of the mouse cardiac troponin I gene. J. Biol. Chem. 269: 339-346.

Baldwin, Jr., A.S., Kittler, E.L., and Emerson, Jr., C.P. 1985. Structure, evolution, and regulation of a fast skeletal muscle troponin I gene. Proc. Natl. Acad. Sci. 82: 8080-8084.

Berrill, N.J. 1955. The origin of vertebrates. Clarendon Press, Oxford, UK

Blumenthal, T. 1995. Trans-splicing and polycistronic transcription in Caenorhabditis elegans. Trends Genet. 11: 132136.

Bonen, L. 1993. Trans-splicing of pre-mRNA in plants, animals, and protists. FASEB J. 7: 40-46

Brehm, K., Jensen, K., and Frosch, M. 2000. mRNA trans-splicing in the human parasitic cestode Echinococcus multilocularis. J. Biol. Chem. 275: 38311-38318.

Bruzik, J.P. and Maniatis, T. 1992. Spliced leader RNAs from lower eukaryotes are trans-spliced in mammalian cells. $\mathrm{Na}$ ture 360: 692-695.

Bruzik, J.P., Van Doren, K., Hirsh, D., and Steitz, J.A. 1988 Trans splicing involves a novel form of small nuclear ribonucleoprotein particles. Nature 335: 559-562.

Campbell, D.A., Thornton, D.A., and Boothroyd, J.C. 1984. Apparent discontinuous transcription of Trypanosoma brucei variant surface antigen genes. Nature 311: 350-355.

Caudevilla, C., Serra, D., Miliar, A., Codony, C., Asins, G., Bach, M., and Hegardt, F.G. 1998. Natural trans-splicing in carnitine octanoyltransferase pre-mRNAs in rat liver. Proc. Nat1. Acad. Sci. 95: 12185-12190.

Conrad, R., Thomas, J., Spieth, J., and Blumenthal, T. 1991. Insertion of part of an intron into the $5^{\prime}$ untranslated region of a Caenorhabditis elegans gene converts it into a transspliced gene. Mol. Cell. Biol. 11: 1921-1926.

Conrad, R., Liou, R.F., and Blumenthal, T. 1993. Conversion of a trans-spliced C. elegans gene into a conventional gene by introduction of a splice donor site. EMBO J. 12: 1249-1255.

Cooper, L.F. and Uoshima, K. 1994. Differential estrogenic regulation of small $\mathrm{M}(\mathrm{r})$ heat shock protein expression in osteoblasts. J. Biol. Chem. 269: 7869-7873.
Corbo, J.C., Levine, M., and Zeller, R.W. 1997. Characterization of a notochord-specific enhancer from the Brachyury promoter region of the ascidian, Ciona intestinalis. Development 124: 589-602.

Corin, S.J., Juhasz, O., Zhu, L., Conley, P., Kedes, L., and Wade, R. 1994. Structure and expression of the human slow twitch skeletal muscle troponin I gene. J. Biol. Chem. 269: 1065110659.

Davis, R.E. 1996. Spliced leader RNA trans-splicing in metazoa. Parasitology Today 12: 33-40.

Edmondson, D.G., Cheng, T.C., Cserjesi, P., Chakraborty, T., and Olson, E.N. 1992. Analysis of the myogenin promoter reveals an indirect pathway for positive autoregulation mediated by the muscle-specific enhancer factor MEF-2. Mol. Cell. Biol. 12: 3665-3677.

Gao, Z. and Garbers, D.L. 1998. Species diversity in the structure of zonadhesin, a sperm-specific membrane protein containing multiple cell adhesion molecule-like domains. $I$. Biol. Chem. 273: 3415-3421.

Hannon, G.J., Maroney, P.A., Yu, Y.T., Hannon, G.E., and Nilsen, T.W. 1992. Interaction of U6 snRNA with a sequence required for function of the nematode SL RNA in trans-splicing. Science 258: 1775-1780.

Hinterberger, T.J., Mays, J.L., and Konieczny, S.F. 1992. Structure and myofiber-specific expression of the rat muscle regulatory gene MRF4. Gene 117: 201-207.

Hinz, M., Moore, M.J., and Bindereif, A. 1996. Domain analysis of human U5 RNA: Cap trimethylation, protein binding, and spliceosome assembly. J. Biol. Chem. 271: 19001-19007.

Imai, K., Satoh, N., and Satou, Y. 1999. Identification and characterization of maternally expressed genes with mRNAs that are segregated with the endoplasm of early ascidian embryos. Int. J. Dev. Biol. 43: 125-133.

Jarmalowski, A. and Mattaj, I.W. 1993. The determinants of Sm protein binding to Xenopus U1 and U5 snRNAs are complex and non-identical. EMBO J. 12: 223-232.

Katz, M.J. 1983. Comparative anatomy of the tunicate tadpole, Ciona intestinalis. Biological Bulletin 164: 1-27.

Kooter, J.M., De Lange, T., and Borst, P. 1984. Discontinuous synthesis of mRNA in trypanosomes. EMBO J. 3:23872392.

Kozak, M. 1991. An analysis of vertebrate mRNA sequences: Intimations of translational control. J. Cell. Biol. 115: 887903.

Kramer, A. 1996. The structure and function of proteins in volved in mammalian pre-mRNA splicing. Annu. Rev. Biochem. 65: 367-409.

Krause, M. and Hirsh, D. 1987. A trans-spliced leader sequence on actin mRNA in C. elegans. Cell 49: 753-761.

Lee, M.G. and Van der Ploeg, L.H. 1997. Transcription of protein-coding genes in trypanosomes by RNA polymerase I. Annu. Rev. Microbiol. 51: 463-489.

Li, B.L., Li, X.L., Duan, Z.J., Lee, O., Lin, S., Ma, Z.M., Chang, C.C., Yang, X.Y., Park, J.P., Mohandas, T.K., et al. 1999. Human acyl-CoA:cholesterol acyltransferase-1 (ACAT-1) gene organization and evidence that the 4.3-kilobase ACAT-1 mRNA is produced from two different chromosomes. J. Biol. Chem. 274: 11060-11071.

MacGregor, G.R., Mogg, A.E., Burke, J.F., and Caskey, C.T. 1987. Histochemical staining of clonal mammalian cell lines expressing E. coli $\beta$ galactosidase indicates heterogeneous expression of the bacterial gene. Somat. Cell Mol. Genet. 13: $253-265$.

MacLean, D.W., Meedel, T.H., and Hastings, K.E.M. 1997. Tissue-specific alternative splicing of ascidian troponin I isoforms. Redesign of a protein isoform-generating mechanism 
during chordate evolution. J. Biol. Chem. 272: 32115-32120.

Mair, G., Shi, H., Li, H., Djikeng, A., Aviles, H.O., Bishop, J.R., Falcone, F.H., Gavrilescu, C., Montgomery, J.L., Santori, M.I., et al. 2000. A new twist in trypanosome RNA metabolism: cis-splicing of pre-mRNA. RNA 6: 163-169.

Marino, R., De Santis, R., Giuliano, P., and Pinto, M.R. 1999. Follicle cell proteasome activity and acid extract from the egg vitelline coat prompt the onset of self-sterility in Ciona intestinalis oocytes. Proc. Natl. Acad. Sci. 96: 9633-9636.

Maroney, P.A., Denker, J.A., Darzynkiewicz, E., Laneve, R., and Nilsen, T.W. 1995. Most mRNAs in the nematode Ascaris lumbricoides are trans-spliced: A role for spliced leader addition in translational efficiency. RNA 1: 714-723.

Mathews, D.H., Sabina, J., Zuker, M., and Turner, D.H. 1999. Expanded sequence dependence of thermodynamic parameters improves prediction of RNA secondary structure. $J$. Mol. Biol. 288: 911-940.

Meedel, T.H. and Hastings, K.E.M. 1993. Striated muscle-type tropomyosin in a chordate smooth muscle, ascidian bodywall muscle. J. Biol. Chem. 268: 6755-6764.

Meedel, T.H., Farmer, S.C., and Lee, J.J. 1997. The single MyoD family gene of Ciona intestinalis encodes two differentially expressed proteins: Implications for the evolution of chordate muscle gene regulation. Development 124: 1711-1721.

Milhausen, M., Nelson, R.G., Sather, S., Selkirk, M., and Agabian, N. 1984. Identification of a small RNA containing the trypanosome spliced leader: A donor of shared 5 ' sequences of trypanosomatid mRNAs? Cell 38: 721-729.

Nilsen, T.W. 1993. Trans-splicing of nematode premessenger RNA. Annu. Rev. Microbiol. 47: 413-440.

Ochman, H., Gerber, A.S., and Hartl, D.L. 1988. Genetic applications of an inverse polymerase chain reaction. Genetics 120: $621-623$.

Rajkovic, A., Davis, R.E., Simonsen, J.N., and Rottman, F.M. 1990. A spliced leader is present on a subset of mRNAs from the human parasite Schistosoma mansoni. Proc. Natl. Acad. Sci. 87:-8883.

Raker, V.A., Hartmuth, K., Kastner, B., and Lührmann, R. 1999. Spliceosomal UsnRNP core assembly: Sm proteins assemble onto an Sm site RNA nonanucleotide in a specific and thermodynamically stable manner. Mol. Cell. Biol. 19: 6554 6565.

Ross, L.H., Freedman, J.H., and Rubin, C.S. 1995. Structure and expression of novel spliced leader RNA genes in Caenorhabditis elegans. J. Biol. Chem. 270: 22066-22075.

Takagi, T., Yasunaga, H., and Nakamura, A. 1993. Structure of $29-\mathrm{kDa}$ protein from ascidian (Halocynthia roretzi) body wall muscle. J. Biochem. 113: 321-326.

Tapscott, S.J., Lassar, A.B., and Weintraub, H. 1992. A novel myoblast enhancer element mediates MyoD transcription. Mol. Cell. Biol. 12: 4994-5003.

Tessier, L.H., Keller, M., Chan, R.L., Fournier, R., Weil, J.H., and Imbault, P. 1991. Short leader sequences may be transferred from small RNAs to pre-mature mRNAs by trans-splicing in Euglena. EMBO I. 10: 2621-2625.

Thomas, P.S. 1980. Hybridization of denatured RNA and small DNA fragments transferred to nitrocellulose. Proc. Natl. Acad. Sci. 77: 5201-5205. 


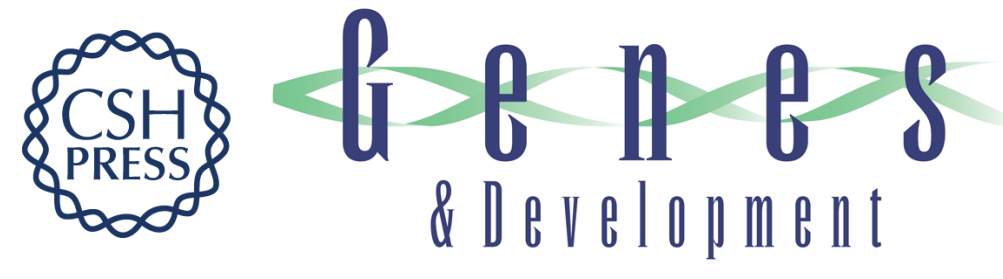

\section{mRNA 5'-leader trans-splicing in the chordates}

Amanda E. Vandenberghe, Thomas H. Meedel and Kenneth E.M. Hastings

Genes Dev. 2001, 15:

Access the most recent version at doi:10.1101/gad.865401

References This article cites 50 articles, 26 of which can be accessed free at: http://genesdev.cshlp.org/content/15/3/294.full.html\#ref-list-1

\section{License}

Email Alerting Receive free email alerts when new articles cite this article - sign up in the box at the top Service right corner of the article or click here.

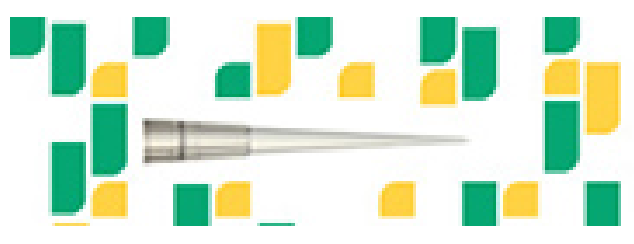

